\title{
ONE MORE METRIZATION THEOREM
}

\author{
H. H. HUNG
}

\begin{abstract}
We give here a metrization theorem proved via the method of symmetrics. From our theorem follow the theorem of Stone-Arhangel'skir and one in terms of a countable strongly refining sequence of open coverings.
\end{abstract}

We propose a new metrization theorem the proof of which uses the method of symmetrics [4], [5], [8]. The new theorem implies Stone-Arhangel' skiir's [1], [4], [9] and that in terms of a countable strongly refining sequence of open coverings [2], [3], [6], [7].

1. Main theorem. We propose the following theorem.

THEOREM 1.1. A $T_{0}$-space $X$ is metrizable if (and only if) there exists a neighbourhood base $\left\{V_{x, n}: n \in \mathbf{N}\right\}$ of $x$ at every $x \in X$ with the following properties.

(i) For all $x, y \in X$ and $n \in \mathbf{N}, x \in V_{y, n} \Leftrightarrow y \in V_{x, n}$.

(ii) For each compact set $K$ in $X$ and each open set $U$ containing $K$, there is such $a$ bounded function $n$ from $K$ into $\mathbf{N}$ that $K \subset \cup_{x \in K} V_{x, n(x)} \subset U$.

Proof (OF THE SUFFICIENCY PART) OF THEOREM 1.1. Given any $T_{0}$-space $X$ and neighbourhood bases of the description in the hypothesis, we can define a nonnegative real valued function $\rho$ on $X \times X$ as follows. For all $x, y \in X, x$ $\neq y$, we can define $\rho(x, y)$ such that $1 / \rho(x, y)=$ smallest $i$ for which $y \notin V_{x, i}$ which is always possible as long as $X$ is $T_{0}$. Indeed $\rho(x, y)=\rho(y, x)$ always. For all $x \in X, \rho(x, x)$ is defined to be 0 . Such a $\rho$ is obviously a symmetric and we refer to it in the following as our symmetric.

To prove that our symmetric induces a sufficiently large topology, it suffices to produce, for every $y \in X$ and every open neighbourhood $A$ of $y$, a ball of some finite radius $r$ centered at $y, N(y, r) \equiv\{x \in X: \rho(x, y)<r\}$, totally within $A$. To prove that the topology so induced is not excessively large and therefore just right, we need only exhibit, for any ball of any (finite) radius about any point, a neighbourhood of the point within that ball. For both our tasks, we need only note that, for all $x \in X, i \in \mathbf{N}, \bigcap_{j \leqslant i, j \in \mathbf{N}} V_{x, j}$ is $N(x, 1 / i)$. The space $X$ can therefore be considered a symmetric space.

If we bring in property (ii) at this juncture, we can plainly see that for any compact set $K$ and any disjoint closed set $C$ on $X, \rho(K, C)>0$. Martin's Theorem [8] and metrizability follow. Q.E.D.

Received by the editors April 17, 1975 and, in revised form, August 8, 1975 and October 18, 1975.

AMS (MOS) subject classifications (1970). Primary 54E35, 54E25.

Key words and phrases. Symmetric spaces, metrization.

O American Mathematical Society 1976 
Corollary 1.2. A T $T_{0}$-space $X$ is metrizable if (and only if) there exists a neighbourhood base $\left\{V_{x, n}: n \in \mathbf{N}\right\}$ of $x$ at every $x \in X$ with the following properties. For each $x \in X$ and $n \in \mathbf{N}$.

(i) $x \in V_{y, n}$ for all $y \in V_{x, n}$,

(ii) there exist such an $m \in \mathbf{N}$ and such an open neighbourhood $W$ of $x$ that $V_{y, m} \subset V_{x, n}$, for all $y \in W$.

Proof (oF THE SUfFiciency ParT) OF Corollary 1.2. Given any $T_{0}$-space $X$ and neighbourhood bases of the description in the hypothesis, we have, for any compact set $K$ and any open set $U$ containing $K$, an open cover $\mathscr{W}=\{W(x): x \in K\}$ of $K$, for every member of which there exists such an $m(x) \in \mathbf{N}$ that $V_{y, m(x)} \subset U$, for all $y \in W(x)$. Because $K$ is compact, there is such a finite subset $F \subset K$ that $\mathfrak{V}=\{W(x): x \in F\}$ is a cover of $K$, and we can define a bounded function $n$ from $K$ into $\mathbf{N}$ of the kind described in (ii) of Theorem 1.1 by the following formula:

$$
n(y)=\min \{m(x): y \in W(x), x \in F\}, \text { for all } y \in K,
$$

and invoke Theorem 1.1. Q.E.D.

2. Applications. We show that our Theorem 1.1 and Corollary 1.2 imply in a very straightforward manner each of the theorems below.

Theorem A (ARhANGel'skiĭ [1], [4], Stone [9]). For a $T_{0}$-space to be metrizable it is necessary and sufficient that there exists on $X$ a countable collection of open coverings $\left\{\mathbb{Q}_{i}\right\}_{i \in \mathbf{N}}$ satisfying the following condition. Given any point $x \in X$ and any neighbourhood $U$ of it, there exist an $i \in \mathbf{N}$ and a (smaller) neighbourhood $V$ of $x$ such that $\mathrm{St}\left(V, \mathbb{Q}_{i}\right) \subset U$.

Theorem B (ARHANGel'skili [2] , 3], F. B. Jones [6], [7]). The class of $\left(T_{0}-\right)$ spaces which have a countable strongly refining sequence of open coverings ${ }^{1}$ coincides precisely with the class of metric spaces.

Proof (of the sufficiency part) of Theorem A. For each $x \in X$ and $i \in \mathbf{N}$, we let St $\left(x, \mathscr{Q}_{i}\right)$ be $V_{x, i}$. Clearly the families $\left\{V_{x, n}: n \in \mathbf{N}\right\}$ so obtained have all the properties described in Corollary 1.2. Indeed if $y \in \operatorname{St}\left(x, \mathbb{Q}_{i}\right)$, there is $A \in \mathbb{Q}_{i}$ in which are both $x$ and $y$ and consequently $x \in \operatorname{St}\left(y, \mathbb{Q}_{i}\right)$. Further, given $x \in X$ and $n \in \mathbf{N}$, there exist an $i \in \mathbf{N}$ and an open neighbourhood $V$ of $x$ such that $\mathrm{St}\left(V, \mathbb{Q}_{i}\right) \subset \mathrm{St}\left(x, \mathbb{Q}_{n}\right)$ and consequently

$$
\text { St }\left(y, \mathbb{Q}_{i}\right) \subset \operatorname{St}\left(x, \mathbb{Q}_{n}\right), \text { for all } y \in V \text {. }
$$

Q. E.D.

(The sufficiency part of) Theorem B can obviously be proved with the proof of Theorem A, except we should invoke Theorem 1.1 rather than its corollary.

\section{REFERENCES}

1. A. V. Arhangel' skiì, New criteria for paracompactness and metrizability of an arbitrary $T_{1^{-}}$

${ }^{1}$ A countable sequence of open coverings $\left\{Q_{i}\right\}_{i \in \mathrm{N}}$ of a space $X$ is said to be strongly refining if for any compact set $K$ and any neighbourhood $U$ of $K$, there is such a $j \in \mathbf{N}$ that $\operatorname{St}\left(K, \mathbb{Q}_{j}\right) \subset U$. 
space, Dokl. Akad. Nauk SSSR 141 (1961), 13-15 = Soviet Math. Dokl. 2 (1961), 1367-1369. MR 24 \# A1113.

2. - Bicompact sets and the topology of spaces, Dokl. Akad. Nauk SSSR 150 (1963), 9-12 = Soviet Math. Dokl. 4 (1963), 561-564. MR 27 \# 720.

3. - Bicompact sets and the topology of spaces, Trudy Moskov. Mat. Obšč. 13 (1965), 3-55 = Trans. Moscow Math. Soc. 13 (1965), 1-62. MR 33 \#3251.

4. - Mappings and spaces, Uspehi Mat. Nauk 21 (1966), no. 4 (130), 133-184 = Russian Math. Surveys 21 (1966), no. 4, 115-162. MR 37 \#3534.

5. H. H. Hung, Some metrization theorems, Proc. Amer. Math. Soc. 54 (1976), 363-367.

6. F. B. Jones, R. L. Moore's axiom l'and metrization, Proc. Amer. Math. Soc. 9 (1958), 487. MR (20) \#278.

7. __ Metrization, Amer. Math. Monthly 73 (1966), 571-576. MR 33 \# 7980.

8. H. W. Martin, Metrization of symmetric spaces and regular maps, Proc. Amer. Math. Soc. 35 (1972), 269-274. MR 46 \#2648.

9. A. H. Stone, Sequences of coverings, Pacific J. Math. 10 (1960), 689-691. MR 22 \#9955.

School of Mathematical Sciences, University of Science of Malaysia, Penang, MalaySIA 